AMS/Industry Undergraduate Scholarships are sponsored by leading environmental science and service corporations and are designed to encourage outstanding undergraduate students to pursue careers in the atmospheric and related oceanic and hydrologic sciences. The awards are $\$ 2000$ for a nine-month period and an additional $\$ 2000$ for a subsequent nine-month period in the senior year, and are available to students who will be juniors in the following academic year. The awards are based on merit and are given to students who have shown the potential for accomplishment in these fields. The Executive Committee selects the awardees based on the recommendations of the Committee of Judges for Undergraduate Awards.

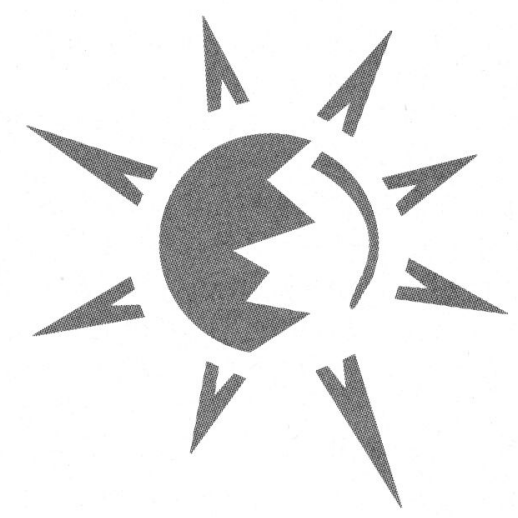

\title{
Statement on Open Meetings
}

All meetings of AMS committees, boards, and the Council are, in principal, open to members of the Society, although portions of some meetings may be held in executive session when dealing with personnel issues, awards, or other matters of a confidential nature.

As a matter of courtesy and to ensure adequately sized meeting rooms, members wishing to observe a particular committee, board, or Council meeting should contact its chairperson in advance. Members may request a place on the agenda by following a similar procedure. Please feel free to contact AMS Headquarters for assistance. 


\section{A. General}

The following general principles apply to Society awards and lectureships.

1. All final decisions on the creation of an award and lectureship-its purposes, terms of reference, and conditionsand on award and lectureship recipients, are made by the AMS Council.

2. Awards and lectureships are to be maintained at the highest level. While an award winner or lecturer may be chosen annually, it may often be more suitable to present the award only occasionally, and an award committee or panel should not hesitate to pass over a year when there is no outstanding nominee. Justification is required if, in an exceptional case, a multiple award is recommended to the Council.

3. With the exception of student awards, awards and lectureships will be solely in the form of medals, plaques, certificates, etc., and not in the form of cash or cash equivalent.

4. All awards and lectureships will be considered for renewal or termination by the Council every eight years beginning 1 January 2000.

\section{B. Establishing Awards/Lectureships}

1. Awards and lectureships are established only when there is clear justification.

2. Awards and lectureships may be named or unnamed.

3. Awards and lectureships are named only for deceased members of the Society who are deserving of such recognition.

4. A proposal for a new award or lectureship may be submitted to the Secretary-Treasurer, American Meteorological Society, 45 Beacon St., Boston, MA 02108 by any member or group of the Society. The proposal, not to exceed five pages in length, shall include sufficient background and justification indicating, for example, that the pool of potential awardees is large enough to justify the new award or lectureship. Additional letters of support from relevant portions of the affected community will be welcome. The proposal shall also include suggested Terms of Reference and the method for selection of awardees.

5. Proposals for a new award or lectureship will be reviewed by the Awards Oversight Committee (AOC). When appropriate, the AOC will request input and comments from other segments of the Society.

6. The AOC will recommend a course of action to the Council.

\section{Award and Lecturer Nomination Procedure}

1. Nominations may be submitted to the Secretary-Treasurer, American Meteorological Society, 45 Beacon St., Boston, MA 02108 by any member or group of the Society. A nomination form is available from AMS Headquarters and for downloading from the AMS Web site.

2. The nomination is to include a proposed citation and a condensed curriculum vitae. Up to three additional letters of support may be submitted with the nomination.

3. Nominations will be active for three years, and updated information may be submitted for consideration in the second and third year.

\section{Selection Procedures}

All awards and lectureships are approved by the Council. The specific procedure for nominating awardees and lecturers is described in the "Policy, Procedures, and Guidelines for Awards and Lectureships."

Recommendations to the Council for awardees and lecturers are made by the various committees and panels described in the relevant Terms of Reference. To improve consistency and avoid overlap and duplication of awards, an AOC was established in 1999. The AOC makes recommendations to the Council for the Brooks, Abbe, Corporation, and Special Awards. Prior to submission to the Council for approval, the AOC also reviews the recommendations for all other awards and lecturers except (1) honorary membership and (2) student awards.

The AOC is chaired by the First Past President and includes as members the President-elect, the President, the Chairs of the Atmospheric Research Awards Committee and the Oceanographic Research Awards Committee, the STAC Com- 
missioner, the Professional Affairs Commissioner, the Education and the Human Resources Commissioner, and the Publications Commissioner.

\section{E. Policy On Named Conferences/Symposia and Special Issues of AMS Journals, as adopted by the Council effective 25 September 1999.}

Recognition of scientists in the fields served by the AMS, living or deceased, in the form of a named conference or symposium or a named special issue of one of the Society's journals is an honor reserved for only the most outstanding of our colleagues. It should be awarded only to those individuals who are completing a career, or who have recently died having completed a career, of significant achievements in their field and whose contributions would make them worthy of consideration for Honorary Member of the AMS.

Proposals for either a named conference/symposium or special issue, including the nature of the content for the conference/symposium or special issue that justifies its naming, should be submitted to the STAC Commissioner or Publications Commissioner, respectively. The Commissioner will utilize the commission membership, with particular weight being given to the input from the affected STAC committee or journal editorial board, to review the proposal and provide recommendations. The Commissioner will forward those proposals deemed appropriate to the Council for approval. Proposals for named conferences and symposia organized outside the STAC structure (such as the IIPS Conference or Education Symposium) will still be handled by the STAC Commissioner, but with considerable input from the organizing committee of the affected meeting and possibly other commissions, committees, or boards as deemed appropriate by the STAC Commissioner. Final approval lies with the AMS Council.

Such awards will be made infrequently, and as a guideline, a particular journal should have an issue named after an individual no more frequently than about once every three years, and a conference/symposium should be named after an individual no more frequently than about once every three meetings for that conference or symposia series. Special symposia that are not part of a series can be organized to honor a scientist.

\section{F. Guidelines for memorials were adopted by the Council on 29 January 1978.}

A memorial is defined as a gift, award, publication, or activity that is accepted or created by action of the Society for the purpose of perpetuating the memory of a person or group of persons. Honorary membership and awards, which are normally bestowed for the purpose of honoring living persons, are not to be construed as memorials under these guidelines.

1. Memorials shall be established by action of the Council.

2. Memorials shall normally be established only for nonliving persons. There shall be no exclusions based on membership in the Society, nationality, race, religion, or sex.

3. Memorials shall satisfy both of the following criteria:

a) The accomplishments and ideals of the person being memorialized shall have been such as to advance the aims of the Society.

b) The objective of the memorial, be it an award, scholarship, publication, or other activity, shall be such as to advance the aims of the Society.

4. Memorials shall be considered in two categories: designated and undesignated.

a) Designated memorials are normally established for a specified object in such a way as to memorialize an individual or group of individuals. If the memorial is in the nature of a periodic payment or award, sufficient capital will normally be provided to fund the award over the stated duration of the memorial.

b) Undesignated memorial gifts may be accepted by the Society to be used in ways and for purposes to be determined by the Council, including the amalgamation of several such memorials for a specific purpose.

5. The designation of memorials to provide scholarships for the assistance of meteorology students at academic institutions is encouraged.

6. The use of memorial funds to publish a special volume or monograph is encouraged. 
1951 Hurd Curtis Willett

1953 Carl-Gustaf Arvid Rossby
1955

1956
Jerome Namias

John von Neumann

\section{The Carl-Gustaf Rossby Award for Extraordinary Scientific Achievement}

(Until 1958, designated as Award for Extraordinary Scientific Achievement)
1960 J. Bjerknes and Erik Palmén
1962 Bernhard Haurwitz
1961 Victor P. Starr
1963 Harry Wexler

\section{The Carl-Gustaf Rossby Research Medal}

(Until 1963, designated as The Carl-Gustaf Rossby Award for Extraordinary Scientific Achievement)

$\begin{array}{llllll}1964 & \text { Jule G. Charney } & 1977 & \text { Akio Arakawa } & 1989 & \text { Richard J. Reed } \\ 1965 & \text { Arnt Eliassen } & 1978 & \text { James W. Deardorff } & 1990 & \text { Yale Mintz } \\ 1966 & \text { Zdenek Sekera } & 1979 & \text { Herbert Riehl } & 1991 & \text { Kikuro Miyakoda } \\ 1967 & \text { Dave Fultz } & 1980 & \text { Sean A. Twomey } & 1992 & \text { Syukuro Manabe } \\ 1968 & \text { Verner E. Suomi } & 1981 & \text { Roscoe R. Braham, Jr. } & 1993 & \text { John M. Wallace } \\ 1969 & \text { Edward N. Lorenz } & 1982 & \text { Cecil E. Leith, Jr. } & 1994 & \text { Jerry D. Mahlman } \\ 1970 & \text { Hsiao-Lan Kuo } & 1983 & \text { Joanne Simpson } & 1995 & \text { Chester W. Newton } \\ 1971 & \text { Norman A. Phillips } & 1984 & \text { Bert R. Bolin } & 1996 & \text { David Atlas } \\ 1972 & \text { Joseph Smagorinsky } & 1985 & \text { Tiruvalam N. } & 1997 & \text { Robert E. Dickinson } \\ 1973 & \text { Christian E. Junge } & & \text { Krishnamurti } & 1998 & \text { Barry Saltzman } \\ 1974 & \text { Heinz H. Lettau } & 1986 & \text { Douglas K. Lilly } & 1999 & \text { Taroh Matsuno } \\ 1975 & \text { Charles H. B. Priestley } & 1987 & \text { Michael E. McIntyre } & 2000 & \text { Susan Solomon } \\ 1976 & \text { Hans A. Panofsky } & 1988 & \text { Brian J. Hoskins } & & \end{array}$

2001 James R. Holton, "for outstanding advances in the dynamics of the stratosphere through theoretical advances, perceptive use of models, and contributions to key measurement programs."

\section{The Second Half Century Award}

\begin{tabular}{|c|c|c|c|c|c|}
\hline \multirow[t]{4}{*}{1970} & Rudolph Hanel, & 1974 & Tiruvalam N. & \multirow[t]{2}{*}{1980} & André J. Robert and \\
\hline & Don T. Hilleary, & & Krishnamurti & & Frederick G. Shuman \\
\hline & Lewis D. Kaplan, and & 1975 & Louis J. Battan & 1981 & Charles D. Keeling \\
\hline & David Q. Wark & 1976 & Roger M. Lhermitte & & Thomas H. Vonder Haar \\
\hline 1972 & Richard J. Reed & 1977 & Syukuro Manabe & 1982 & James R. Holton \\
\hline 973 & Douglas K. Lilly & 1978 & Joost A. Businger & & Hans R. Pruppacher \\
\hline 1974 & James W. Deardorff & 1979 & J. Murray Mitchell, Jr. & & \\
\hline
\end{tabular}


The Jule G. Charney Award

(Until 1982, designated as Second Half Century Award)

$\begin{array}{llllll}1983 & \text { Francis P. Bretherton } & 1989 & \text { Eugene M. Rasmusson } & 1994 & \text { William M. Gray } \\ 1984 & \text { Peter V. Hobbs } & 1990 & \text { Melvin A. Shapiro } & 1995 & \text { Eugenia Kalnay } \\ 1985 & \text { Keith A. Browning } & & \text { Peter J. Webster } & 1996 & \text { Yoshio Kurihara } \\ & \text { Richard S. Lindzen } & 1991 & \text { Moustafa T. Chahine } & 1997 & \text { Timothy N. Palmer } \\ 1986 & \text { Michio Yanai } & & \text { M. Patrick McCormick } & 1998 & \text { Kuo-Nan Liou } \\ 1987 & \text { Richard A. Anthes } & 1992 & \text { Lance F. Bosart } & 1999 & \text { Anthony Hollingsworth } \\ 1988 & \text { Robert E. Dickinson } & 1993 & \text { Abraham H. Oort } & 2000 & \text { Kevin E. Trenberth }\end{array}$

2001 Roger Daley, "for highly significant research and development in data assimilation, modeling, and numerical weather prediction."

\section{The Verner E. Suomi Award}
1997 John E. Hart
1998 William L. Smith
2000 Michael D. King

2001 Daniel Rosenfeld, "for key contributions to remote measurement and interpretation of rainfall, cloud optical properties, and cloud microphysical properties."

\section{The Sverdrup Gold Medal}

$\begin{array}{llllll}1964 & \text { Henry Stommel } & 1981 & \text { Jerome Namias } & 1993 & \text { Tim P. Barnett } \\ 1966 & \text { Walter H. Munk } & 1983 & \text { Michael S. } & 1994 & \text { Mark A. Donelan } \\ 1970 & \text { Kirk Bryan } & & \text { Longuet-Higgins } & 1995 & \text { James F. Price } \\ 1971 & \text { Klaus Hasselmann } & 1985 & \text { S. George Philander } & 1996 & \text { Julian P. McCreary, Jr. } \\ 1972 & \text { Vladimir Kamenkovich } & 1987 & \text { James J. O’Brien } & 1997 & \text { Kristina B. Katsaros } \\ 1975 & \text { Owen M. Phillips } & 1988 & \text { Hisashi Mitsuyasu } & 1998 & \text { Willard J. Pierson } \\ 1976 & \text { Robert W. Stewart } & 1991 & \text { Klaus Wyrtki } & 1999 & \text { John Stuart Godfrey } \\ 1977 & \text { Raymond B. Montgomery } & 1992 & \text { Mark A. Cane } & 2000 & \text { Mojib Latif } \\ & \text { John C. Swallow } & & & & \end{array}$

2001 Stefan Hastenrath, "for numerous insightful and fundamental contributions to the description and diagnosis of large-scale ocean-atmosphere interactions."

\section{The Henry Stommel Research Award}

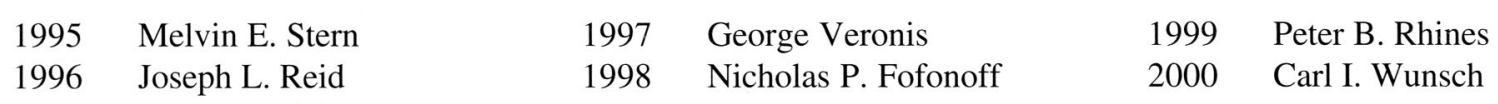

2001 Christopher J. R. Garrett, "for his rare ability to use simple models or concepts to expose the rich underlying physics that leads us all to a more profound understanding of ocean processes." 
1951 Henry Southworth Shaw

1953 Harry Guggenheim
1955 Charles Franklin Brooks

1956 Robert Granville Stone
1957

1958
Carl-Gustaf Arvid Rossby

Henry Garrett Houghton

\section{The Charles Franklin Brooks Award for Outstanding Services to the Society}

(Until 1958, designated Award for Outstanding Services to the Society)

$\begin{array}{llllll}1960 & \text { Horace Robert Byers } & 1973 & \text { Glenn R. Hilst } & 1988 & \text { Evelyn Mazur } \\ 1961 & \text { Howard T. Orville } & 1974 & \text { David F. Landrigan } & 1989 & \text { Kenneth C. Spengler } \\ 1962 & \text { Sverre Petterssen } & 1975 & \text { Werner A. Baum } & 1990 & \text { Eugene W. Bierly } \\ 1963 & \text { David M. Ludlum } & 1976 & \text { Earl G. Droessler } & 1991 & \text { Joseph Smagorinsky } \\ 1964 & \text { Thomas F. Malone } & 1977 & \text { Eugene Bollay } & 1992 & \text { Joanne Simpson } \\ 1965 & \text { Patrick D. McTaggart- } & 1979 & \text { Robert M. White } & 1993 & \text { George S. Benton } \\ & \text { Cowan } & 1980 & \text { Verner E. Suomi } & 1994 & \text { Richard E. Orville } \\ 1966 & \text { John C. Beckman } & 1981 & \text { Charles L. Hosler, Jr. } & 1995 & \text { Harold D. Orville } \\ 1967 & \text { Phil E. Church } & 1982 & \text { David S. Johnson } & 1996 & \text { Eli J. Katz } \\ 1968 & \text { Kenneth C. Spengler } & 1983 & \text { Chester W. Newton } & 1997 & \text { Robert T. Ryan } \\ 1969 & \text { Alfred K. Blackadar } & 1984 & \text { Richard J. Reed } & 1998 & \text { Donald R. Johnson } \\ 1970 & \text { Robert Dawson Fletcher } & 1985 & \text { Robert G. Fleagle } & 1999 & \text { Robert L. Carnahan } \\ 1971 & \text { Louis J. Battan } & 1986 & \text { Richard E. Hallgren } & 2000 & \text { David D. Houghton } \\ 1972 & \text { Helmut E. Landsberg } & 1987 & \text { Roscoe R. Braham, Jr. } & & \end{array}$

2001 Dayton G. Vincent, "for outstanding services to the Society over many years particularly in the areas of tropical meteorology and the meteorology of the Southern Hemisphere."

\section{The Cleveland Abbe Award for Distinguished Service to Atmospheric Sciences by an Individual}

\begin{tabular}{|c|c|c|c|c|c|}
\hline 1963 & Lloyd V. Berkner & 1977 & Richard M. Goody & 1989 & George J. Haltiner \\
\hline 1964 & Francis W. Reichelderfer & 1978 & Horace R. Byers & & Edwin Kessler \\
\hline 1965 & Sverre Petterssen & 1980 & Jule G. Charney and & 1990 & Vincent E. Lally \\
\hline 1966 & Alan T. Waterman & & Joseph Smagorinsky & 1991 & Robert H. Simpson \\
\hline 1967 & Arthur F. Merewether & 1981 & Stanley A. Changnon, Jr. & 1992 & Earl G. Droessler \\
\hline 1968 & Thomas F. Malone & 1982 & Henry G. Houghton & 1993 & Kenneth C. Spengler \\
\hline 1969 & Robert M. White & & Joachim P. Kuettner & 1994 & Francis P. Bretherton \\
\hline 1970 & Walter Orr Roberts & 1983 & Helmut E. Landsberg & 1995 & Glenn R. Hilst \\
\hline 1971 & Robert G. Fleagle & & David Atlas & 1996 & Ronald C. Taylor \\
\hline 1972 & Homer E. Newell & 1984 & C. Gordon Little & 1997 & James R. Mahoney \\
\hline 1973 & Fred D. White & 1985 & Sir Arthur Davies & 1998 & Elbert W. Friday, Jr. \\
\hline 1974 & Lester Machta & 1986 & Roy L. Jenne & 1999 & J. Michael Hall \\
\hline 1975 & George P. Cressman & 1987 & Alfred K. Blackadar & 2000 & Eugene W. Bierly \\
\hline 1976 & Patrick D. McTaggart- & 1988 & Werner A. Baum & & \\
\hline
\end{tabular}

2001 Richard E. Carbone, "for building consensus in the weather research community on problems of major national and international importance, and for fostering the conduct of collaborative and coordinated weather research." 


\section{The Charles E. Anderson Award}

2000 Warren M. Washington

2001 Joanne Simpson, "for her outstanding efforts in promoting diversity within the Society and the greater scientific community over decades, through her commitment to mentoring young professionals and guiding them toward successful careers."

\section{The Clarence Leroy Meisinger Award}

$\begin{array}{rlrlrl}1938 & \text { Jerome Namias } & 1966 & \begin{array}{l}\text { George W. Platzman } \\ \text { Tetsuya Fujita }\end{array} & 1982 & \text { Robert A. Houze, Jr. } \\ 1941 & \text { Joseph J. George } & 1967 & 1983 & \begin{array}{l}\text { Chih-Pei Chang } \\ \text { Joseph Smagorinsky, }\end{array} \\ 1946 & \text { Morris Neiburger } & & & \text { Joseph B. Klemp and } \\ 1947 & \text { Herbert Riehl } & & \text { Syukuro Manabe, } & & \text { Robert B. Wilhelmson } \\ 1948 & \text { James E. Miller } & & \text { Yale Mintz, } & 1984 & \text { Robert A. Maddox } \\ 1949 & \text { Jule G. Charney and } & & \text { Akio Arakawa, and } & 1985 & \text { Louis W. Uccellini } \\ & \text { Arnt Eliassen } & & \text { Cecil E. Leith } & 1986 & \text { Kerry A. Emanuel } \\ 1950 & \text { John Freeman, Jr. and } & 1968 & \text { Katsuyuki Ooyama } & 1987 & \text { Isaac M. Held } \\ & \text { Morris Tepper } & 1969 & \text { Richard S. Lindzen } & 1988 & \text { William K. M. Lau } \\ 1951 & \text { Dave Fultz } & 1970 & \text { William L. Smith } & 1989 & \text { Daniel Keyser } \\ 1956 & \text { Ernest J. Fawbush and } & 1971 & \text { Joseph Pedlosky } & 1990 & \text { Ngar-Cheung Lau } \\ & \text { Robert C. Miller } & 1972 & \text { Francis P. Bretherton } & 1991 & \text { Da-Lin Zhang } \\ 1957 & \text { David Atlas } & 1973 & \text { Robert E. Dickinson } & 1992 & \text { Roger M. Wakimoto } \\ 1959 & \text { Robert G. Fleagle } & & \text { James R. Holton } & 1993 & \text { John C. Derber } \\ 1960 & \text { Philip D. Thompson and } & 1974 & \text { Keith A. Browning } & & \text { David J. Karoly } \\ & \text { Norman A. Phillips } & 1975 & \text { John M. Wallace } & 1994 & \text { David A. Randall } \\ 1961 & \text { Verner E. Suomi } & 1976 & \text { Thomas W. Flattery } & 1995 & \text { Kevin P. Hamilton } \\ 1962 & \text { Louis J. Battan } & 1977 & \text { Roger A. Pielke } & 1996 & \text { J. David Neelin } \\ & \text { Joanne Starr Malkus } & 1978 & \text { Alan K. Betts } & 1997 & \text { Chin-Hoh Moeng } \\ 1963 & \text { Edward N. Lorenz } & 1979 & \text { John C. Wyngaard } & 1998 & \text { David J. Stensrud } \\ 1964 & \text { Richard J. Reed } & 1980 & \text { Richard A. Anthes } & 1999 & \text { Clara Deser } \\ 1965 & \text { Hans A. Panofsky } & 1981 & \text { J. Michael Fritsch } & 2000 & \text { Karen Hepler Rosenlof }\end{array}$

2001 James W. Hurrel, "for his authoritative, lucid, and elegant analysis of the North Atlantic oscillation and of recent measurements of tropospheric temperature trends."

\section{The Henry G. Houghton Award}

$\begin{array}{llllll}1990 & \text { Graeme L. Stephens } & 1994 & \text { Venkatachalam } & 1997 & \text { Piers J. Sellers } \\ 1991 & \text { Susan Solomon } & & \text { Ramaswamy } & 1998 & \text { Patrick Minnis } \\ 1992 & \text { Judith A. Curry } & 1995 & \text { Bruce A. Wielicki } & 1999 & \text { Michael I. Mishchenko } \\ 1993 & \text { Harshvardhan } & 1996 & \text { David W. Fahey } & 2000 & \text { William H. Brune }\end{array}$

2001 K. Franklin Evans, "for perceptive conceptual advances in theory, measurement, and three-dimensional modeling of radiative transfer in cloudy skies." 
The Award for Outstanding Service by a Weather Forecaster

$\begin{array}{ll}1967 & \text { Charles L. Mitchell } \\ 1968 & \text { Gordon E. Dunn } \\ 1969 & \text { Lee George Dickinson } \\ 1970 & \text { Harlan K. Saylor } \\ 1971 & \text { Leonard W. Snellman } \\ 1972 & \text { Robert E. Clark } \\ 1973 & \text { Hilmer Crumrine }\end{array}$

1967

1969

1970

1972

1973

\author{
1974 \\ 1975 \\ 1976 \\ 1977 \\ 1978
}
1979 Robert J. C. Burnash and Arthur F. Gustafson
1980 Vernon G. Bohl
1981 Gilbert B. Clark
Paul H. Swope
1982 John P. Crozier

\section{The Francis W. Reichelderfer Award}

(Until 1982, designated as Award for Outstanding Service by a Weather Forecaster)
1983 John R. Hope
1985
Alan R. Moller
1984 James D. Belville, Gary K. Grice,
1986 G. Alan Johnson

and Jimmy D. Ward

(Until 1986, for Long-Term Service by Persons Engaged in Weather Forecasting Activities)

(After 1986, awarded for Distinguished Public Contributions by Personnel of the Weather Services)

\begin{tabular}{llclrl}
1987 & Robert E. Muller & 1992 & Judson W. Ladd & 1996 & Max Mayfield \\
1988 & Fredrich Galster and & 1993 & Thomas A. Niziol & 1997 & Gregory E. Jackson \\
& William J. Gery & 1994 & Glenn S. Austin and & 1998 & Lans P. Rothfusz \\
1989 & Stanley E. Wasserman & & Rodney F. Gonski & 1999 & Roderick A. Scofield \\
1990 & Phillip D. Bothwell & \multirow{2}{*}{1995} & Vernon E. Kousky & 2000 & Scott Plischke \\
1991 & Joel D. Martin & & & &
\end{tabular}

2001 Xavier William Proenza, "for exceptional dedication to public service and effective long-term leadership aimed at producing the highest level of warning and forecast operations at National Weather Service offices."

\section{The Charles L. Mitchell Award}

(Since 1982, awarded for an Exceptional Specific Prediction)

$\begin{array}{llll}1983 & \text { Ronald K. Surface and Jerry A. Youngberg } & 1986 & \text { John E. Hales, Jr., Charles D. Little, and } \\ 1984 & \text { Jack Hollis } & \text { James M. Lowe } \\ 1985 & \text { Robert E. Fischer } & \end{array}$

(Until 1987, awarded for an Exceptional Specific Prediction)

(After 1987, awarded for Long-Term Service by Persons Engaged in Weather Forecasting Activities)

$\begin{array}{llllll}1987 & \text { John A. Brown, Jr. } & 1991 & \text { Donald R. Devore } & 1996 & \text { Gene J. Pfeffer } \\ & \text { Leo C. Clarke } & 1992 & \text { Marvin E. Miller } & 1997 & \text { Kermit K. Keeter } \\ 1988 & \text { Gifford F. Ely, Jr. } & 1993 & \text { Glenn D. Hamilton } & 1998 & \text { Anthony G. Barnston } \\ 1989 & \text { Gilbert B. Clark } & 1994 & \text { Elliot Abrams } & 1999 & \text { Robert W. Burpee } \\ 1990 & \text { George W. Cry } & 1995 & \text { John D. Stackpole } & 2000 & \text { Bradley R. Colman }\end{array}$




\begin{tabular}{|c|c|c|c|c|c|}
\hline 1987 & James A. Kaplan & 1993 & Walter H. Drag & 1999 & Anthony J. Cristaldi III, \\
\hline & John R. Roadcap & 1994 & Henry E. Buehner & & Bartlett C. Hagemeyer, \\
\hline 1988 & Larry H. Eblen & 1995 & Robert S. Davis and & & David W. Sharp, and \\
\hline 1989 & Eddie G. Brite, & & Roger Stairs & & Scott M. Spratt \\
\hline & $\begin{array}{l}\text { Constantine Pashos, } \\
\text { Patrick Tyra, and }\end{array}$ & 1996 & $\begin{array}{l}\text { Andrew D. Stern and } \\
\text { James L. Wiesmueller }\end{array}$ & 2000 & $\begin{array}{l}\text { David R. Sanders and } \\
\text { Peter J. Wilensky }\end{array}$ \\
\hline 1990 & $\begin{array}{l}\text { John V. Wright, Jr. } \\
\text { Larry F. Wilson }\end{array}$ & 1997 & $\begin{array}{l}\text { Ira Kosovitz, } \\
\text { Bruce C. Renneke, and }\end{array}$ & & \\
\hline 1991 & Norman W. Junker & & David R. Willson & & \\
\hline 1992 & Edward R. Agre & 1998 & Andrew D. Stern & & \\
\hline
\end{tabular}

2001 Stephen F. Corfidi, "for his exceptional forecast of the Jarrell, Texas, tornado on Tuesday, 27 May 1997."

\section{The Award for Outstanding Contribution to the Advance of Applied Meteorology}

$\begin{array}{llrlrl}1956 & \text { Joseph J. George } & 1971 & \text { George P. Cressman } & 1985 & \text { E. Philip Krider } \\ 1957 & \text { Vincent J. Schaefer } & 1972 & \text { Vincent J. Oliver } & 1986 & \text { Dennis W. Trettel } \\ 1959 & \text { Carl-Gustaf Arvid } & & \text { Howard B. Kaster } & 1988 & \text { T. Theodore Fujita } \\ & \text { Rossby, posthumously } & 1973 & \text { Harold A. Bedient } & 1989 & \text { Norman A. Phillips } \\ 1960 & \text { Henry T. Harrison } & & \text { Robert E. Munn } & 1990 & \text { Franklin A. Gifford, Jr. } \\ 1961 & \text { Robert D. Elliott } & 1974 & \text { Robert A. McCormick } & 1991 & \text { Stanley A. Changnon, Jr. } \\ 1962 & \text { Alfred H. Glenn } & 1975 & \text { William H. Klein } & 1992 & \text { Michael R. Smith } \\ 1963 & \text { Herbert C. S. Thom } & 1976 & \text { Don G. Friedman } & 1993 & \text { Richard H. Thuillier } \\ 1965 & \text { Loren W. Crow } & & \text { Bernard Vonnegut } & 1994 & \text { Steven R. Hanna } \\ 1966 & \text { Eugene Bollay } & 1977 & \text { John E. Wallace } & 1995 & \text { Keith J. Brown } \\ 1967 & \text { Charles Pennypacker } & 1980 & \text { Allan H. Murphy } & 1996 & \text { Hal W. Brown } \\ & \text { Smith } & 1981 & \text { Harry R. Glahn } & 1997 & \text { Leslie R. Lemon } \\ 1968 & \text { Wallace E. Howell } & 1982 & \text { J. Stewart Marshall } & 1998 & \text { George E. McVehil } \\ 1969 & \text { E. Wendell Hewson } & 1983 & \text { Charles J. Neumann } & 1999 & \text { Peter R. Leavitt } \\ 1970 & \text { Arthur F. Merewether } & 1984 & \text { Paul B. MacCready } & 2000 & \text { Fred V. Brock }\end{array}$

2001 William H. Haggard, "for a long and distinguished career in advancing the field of applied meteorology in both the public and private sector in climatology and forensic meteorology."

\section{The Award for Outstanding Service by a Broadcast Meteorologist}

$\begin{array}{llllll}1977 & \text { Roy L. Leep, Jr. } & 1986 & \text { James D. Williams } & 1993 & \text { Elliot Abrams } \\ 1980 & \text { Donald E. Kent } & 1987 & \text { Richard R. Fletcher } & 1994 & \text { Bruce W. Schwoegler } \\ 1981 & \text { Conrad L. Johnson } & 1988 & \text { Raymond E. Falconer } & 1995 & \text { Kirk T. Melhuish } \\ 1982 & \text { Harry A. Volkman } & 1989 & \text { Jack C. Capell } & 1996 & \text { Leonard M. Slesick } \\ 1983 & \text { John S. Coleman } & 1990 & \text { Terrence F. Kelly } & 1997 & \text { Thomas E. Skilling III } \\ 1984 & \text { George A. Winterling } & 1991 & \text { Peter F. Giddings } & 1998 & \text { Troy M. Kimmel, Jr. } \\ 1985 & \text { Gregory B. Fishel and } & 1992 & \text { Harold E. Taft, } & 1999 & \text { Harvey Leonard } \\ & \text { William Schmidt } & & \text { posthumously } & 2000 & \text { John B. McLaughlin }\end{array}$

2001 Paul S. Joseph, "for his pioneering efforts in broadcast meteorology serving the Milwaukee television and radio market for nearly 30 years." 
The Award for Outstanding Achievement in Bioclimatology

$\begin{array}{llllll}1960 & \text { Frederick Sargent II } & 1969 & \text { William G. Wellington } & 1978 & \text { Norman J. Rosenberg } \\ 1963 & \text { Konrad J. K. Buettner } & 1971 & \text { David M. Gates } & 1980 & \text { Champ B. Tanner } \\ 1964 & \text { Helmut E. Landsberg } & 1972 & \text { Igho H. Kornblueh } & 1981 & \text { William E. Reifsnyder } \\ 1966 & \text { Frederick A. Brooks } & 1973 & \text { Harold D. Johnson } & 1982 & \text { Harold C. Fritts } \\ 1967 & \text { Paul E. Waggoner } & 1976 & \text { G. LeRoy Hahn } & & \end{array}$

\section{The Award for Outstanding Achievement in Biometeorology}

(Until 1982, designated as Award for Outstanding Achievement in Bioclimatology)

\begin{tabular}{|c|c|c|c|c|}
\hline Wilbert O. Haufe & 1990 & Edgar R. Lemon & 1997 & Gerd Jendritzky \\
\hline Robert H. Shaw & 1992 & Donald E. Aylor & 1998 & William P. Lowry \\
\hline Helmut Leith & 1996 & Michael E. Irwin & 1999 & Robert E. Munn \\
\hline
\end{tabular}

\section{The Award for Outstanding Services to Meteorology by a Corporation}

\begin{tabular}{|c|c|}
\hline 1951 & House Beautiful (magazine) \\
\hline 1953 & The Munitalp Foundation \\
\hline 1954 & National Broadcasting Corporation \\
\hline 1955 & Science Service Inc. \\
\hline 1956 & The New York Times \\
\hline 1957 & The Travelers Insurance Companies \\
\hline 1959 & General Electric Company \\
\hline \multirow[t]{3}{*}{1960} & American Airlines, Inc., Eastern Air Lines, \\
\hline & Pan American World Airways, Inc., Trans \\
\hline & World Airlines, and United Air Lines \\
\hline 1961 & Pacific Gas and Electric Company \\
\hline 1963 & Radio Corporation of America \\
\hline 1965 & The Christian Science Monitor \\
\hline \multirow[t]{2}{*}{1966} & Industrial Laboratories of the International \\
\hline & Telephone and Telegraph Corporation \\
\hline 1967 & Atlantic Research Corporation \\
\hline \multirow[t]{2}{*}{1968} & Santa Barbara Research Center of Hughes \\
\hline & Aircraft Company \\
\hline 1969 & Science Associates, Inc. \\
\hline \multirow[t]{4}{*}{1970} & A. H. Glenn \& Associates, Murray and \\
\hline & Trettel, Inc., North American Weather \\
\hline & Consultants, Northeast Weather Service, and \\
\hline & Weather Corporation of America \\
\hline 1971 & Scientific American \\
\hline 1972 & General Electric Company \\
\hline 1973 & WTVT Television Service of Tampa, Fla. \\
\hline 1974 & Barnes Engineering Company \\
\hline 1975 & National Geographic Society \\
\hline 1976 & The Franklin Institute \\
\hline
\end{tabular}

University Corporation for Atmospheric
Research

Massachusetts Institute of Technology

1979 Environmental Research and Technology, Inc.

1980 Helen Dwight Reid Educational Foundation

(HELDREF)

1981 Particle Measuring Systems

1982 Maryland Center for Public Broadcasting

1983 Academic Press, Inc.

1984 Electric Power Research Institute

1985 SRI International

1986 Gannet Co., Inc.

1987 For Spacious Skies Foundation

1988 National Wildlife Federation

1989 General Motors Research Laboratories

1990 The Weather Channel

1991 WSI Corporation

992 Alden Electronics, Inc.

1993 Atmospheric and Environmental Research, Inc.

1994 Electric Power Research Institute

1995 Mount Washington Observatory

1996 Cray Research, Inc.

1997 Space Systems/Loral

1998 R. M. Young Company

1999 Hughes Aircraft Company/Hughes Space and Communications Company and Raytheon Systems Company

WeatherData Incorporated

2001 Cambridge University Press, "for its support of research and teaching in the atmospheric and related sciences through the publication of important monographs and texts." 


\section{The Editor's Awards}

\begin{tabular}{|c|c|c|c|}
\hline 1969 & Norman A. Phillips & 1990 & William Blumen \\
\hline 1970 & Chester W. Newton & & Andrew N. Staniforth \\
\hline 1971 & Peter V. Hobbs & & Robert E. Livezey \\
\hline 1972 & James W. Deardorff & & Dennis H. McCarthy \\
\hline 1973 & George W. Platzman & 1991 & John M. Brown \\
\hline 1974 & Norihiko Fukuta & & Peter Müller \\
\hline 1975 & Gabriel T. Csanady & & Norman W. Junker \\
\hline \multirow[t]{2}{*}{1976} & Stanley L. Barnes & 1992 & Bradley F. Smull \\
\hline & Robert E. Dickinson & & Paul L. Smith \\
\hline 1978 & Jerry D. Mahlman & & Ann E. Gargett \\
\hline 1979 & John M. Wallace & & John R. Gyakum \\
\hline 1980 & Kenneth H. Bergman & & Stuart D. Smith \\
\hline 1981 & Julian P. McCreary, Jr. & & John E. Walsh \\
\hline 1982 & Robert A. Houze, Jr. & 1993 & Dennis L. Hartmann \\
\hline 1983 & Roland A. Madden & & Paola Malanotte-Rizzoli \\
\hline 1984 & Arnold Court & & Philip J. Rasch \\
\hline 1985 & Kenneth H. Brink & & Dusan S. Zrnic \\
\hline \multirow[t]{5}{*}{1986} & Robert P. Davies-Jones & & Joseph T. Schaefer \\
\hline & Michael B. Richman & & David S. Gutzler \\
\hline & Dean H. Roemich & 1994 & Chin-Hoh Moeng \\
\hline & Ronald B. Smith & & Carmen J. Nappo \\
\hline & Alvin L. Morris & & John M. Huthnance \\
\hline \multirow[t]{5}{*}{1987} & Greg J. Holland and & & Marilyn M. Wolfson \\
\hline & John L. McBride & & Albert J. Plueddemann \\
\hline & Edwin L. Crow & & Charles A. Doswell III \\
\hline & Andrew F. Bennett & & John R. Lanzante \\
\hline & James E. Dye & 1995 & Marvin A. Geller \\
\hline \multirow[t]{4}{*}{1988} & James R. Holton & & Witold F. Krajewski \\
\hline & Thomas R. Karl & & William G. Large \\
\hline & Glenn R. Ierley & & Michael L. Kaplan \\
\hline & William A. Cooper & & Robert E. Cheney \\
\hline \multirow[t]{5}{*}{1989} & Margaret A. Lemone & & Paul J. Kocin \\
\hline & Gary A. Briggs & & Richard D. Rosen \\
\hline & Daniel Keyser & & \\
\hline & Andrew J. Weinheimer & & \\
\hline & Kevin E. Trenberth & & \\
\hline
\end{tabular}

1996 Qin Xu

Jeremy M. Hales

Edward S. Sarachik

Richard E. Orville

Dudley B. Chelton

J. Paul Dallavalle

Dennis L. Hartmann

1997 David J. Raymond

Grant W. Petty

Edward C. Monahan

John W. Nielsen-Gammon

Walter F. Dabberdt

Allan H. Murphy

Clara Deser

1998 Brian E. Mapes

Ramesh C. Srivastava

Claes G. Rooth

Kevin R. Knupp

Lucy R. Wyatt

Stephen F. Corfidi

Dennis L. Hartmann

1999 Xubin Zeng

Francis L. Ludwig

Seelye Martin

Edward J. Zipser

R. Paul Lawson

Warren Blier

John E. Walsh

2000 David L. Mitchell

Marvin L. Wesely

Frank S. Henyey

Colleen A. Leary

Luc R. Bissonnette

Adrian Marroquin

Gerald A. Meehl

2001 Bjorn B. Stevens (Journal of the Atmospheric Sciences), "for many thoughtful reviews that have helped add scholarly perspective to papers."

Rod Frehlich (Journal of Applied Meteorology), "for exceptional efforts in making very professional and clear suggestions for the improvement of manuscripts."

Jerome A. Smith (Journal of Physical Oceanography), "for providing exceptionally meritorious reviews of manuscripts."

David M. Schultz (Monthly Weather Review), "for providing extremely thorough, timely, and constructive evaluations of a large number of manuscripts over a diverse range of topics, and for special assistance to the editors in evaluating controversial issues."

Craig J. Donlan (Journal of Atmospheric and Oceanic Technology), "for his insightful reviews." 
Steven J. Weiss (Weather and Forecasting), "for the completion of several extremely knowledgeable, very constructive, and remarkably thorough reviews for manuscripts focused on severe weather detection and prediction."

Song Yang (Journal of Climate), "for providing multiple, prompt, comprehensive, and thoughtful reviews."

Katherine K. Hirschboek (Journal of Hydrometeorology), "for exceptionally thorough reviews of papers submitted during the journal's inaugural year."

\section{The Outstanding Chapter Award}

2001 Omaha-Offut Chapter (Local Chapter), "for Outstanding service to their members, the Society, and their local community including exemplary mentoring of youth.

Lyndon State Student Chapter (Student Chapter), "for exceptional interaction with the professional community, and outstanding service to their members and the Society."

\section{The Robert E. Horton Lecturer in Hydrology}

$\begin{array}{llllll}1974 & \text { Luna B. Leopold } & 1982 & \text { Ray K. Linsley } & 1993 & \text { Wilfried H. Brutsaert } \\ 1975 & \text { Max A. Kohler } & 1983 & \text { Robert J. C. Burnash } & 1995 & \text { Ignacio Rodriguez-Iturbe } \\ 1976 & \text { William C. Ackermann } & 1985 & \text { Floyd A. Huff } & 1997 & \text { Eugene M. Rasmusson } \\ 1977 & \text { James P. Bruce } & 1988 & \text { Peter S. Eagleson } & 1999 & \text { Rafael L. Bras } \\ 1980 & \text { Eugene L. Peck } & 1991 & \text { James C. I. Dooge } & & \end{array}$

2001 Eric F. Wood, "for pioneering analyses of the scaling properties of land surface processes and landatmosphere interactions."

\section{The Bernhard Haurwitz Memorial Lecturer}
1989 John M. Wallace
1996
Brian J. Hoskins
1999
Isaac M. Held
1993 James R. Holton
1997 Richard S. Lindzen
2000
Conway B. Leovy

2001 R. Alan Plumb, "for major contributions to understanding stratospheric transport and the quasi-biennial oscillation."

\section{The Remote Sensing Lecturer}
1991
David Atlas
1997
Edgeworth R. Westwater
1999
S. Harvey Melfi
1993 Verner E. Suomi
1998
William L. Smith
2000
M. Patrick McCormick
1996 Richard M. Schotland

2001 Robert D. Cess, "for pioneering contriburions to the development and application of remote sensing for understanding the earth's radiation budget." 


\section{The Walter Orr Roberts Memorial Lecturer in Interdisciplinary Sciences}
1991 John E. Kutzbach
1996
Duncan C. Blanchard
Syukuro Manabe
1998 Bruce B. Hicks
1999 Richard C. J. Somerville
2000 Robert E. (Ted) Munn

2001 Jonathan T. Overpeck, "for outstanding contributions to the field of paleoclimatology and leadership in fostering Earth system science."

\section{Special Awards and Citations}

1957 Radio Station KSOK of Arkansas City, Kansas Television Station WKY-TV of Oklahoma City, Oklahoma

Joseph Bartatto

Stuart Grazier Bigler

1959

Maurice Levy

Jean Felix Piccard

1960 U.S. Forest Service

Charles B. Moore, Malcolm D. Ross, and, posthumously, Lee Lewis

Walter Rue

Nicholas Brango

1961 William W. Kellogg and Stanley M. Greenfield John C. Freeman and Archie M. Kahan

1962 Werner A. Baum

1964 Dean Blake

Reverend Adelhelm Hess, O.S.B.

1965 Gordon D. Cartwright and Morton J. Rubin

1966 Gertrude M. Woods

1969 Fortune Magazine and Lawrence Lessing

Station KICD, Spencer, Iowa

1970 Hydrologic Services of the ESSA Weather

Bureau

Ferdinand C. Bates, posthumously

Howard H. Hanks, Jr., posthumously

WMAQ-TV, Chicago, Illinois

1971 George L. Hammond

Robert E. Cardinal

National Broadcasting Company

WKY Television System, Incorporated, of

Oklahoma City

Raymond E. Falconer and

Raymond A. Wrightson

1972 Robert Jastrow

Francis W. Reichelderfer

Malcolm Rigby

1973 Agricultural (Fruit Frost) Weather Forecasters in Arizona and California

William H. Best, Jr.

James W. Reid, posthumously

1974 Illinois State Water Survey
1975 Robert O. Reid

National Weather Service Office at

Huntsville, Alabama

Det. 15, 15th Wea. Sq., 5th Wea. Wg., Air Weather Service, Wright-Patterson AFB, Ohio

1976 Albert W. Duckworth

Vincent J. Schaefer

1977 Viking Meteorology Flight Team: Seymour

L. Hess, Team Leader, Florida State University; Jack A. Ryan, Deputy Team Leader,

California State University at Fullerton;

Thomas E. Chamberlain, TRW Defense and Space Systems Group; Harold L. Cole,

National Center for Atmospheric Research;

Richard G. Dutton, Martin Marietta Aero-

space; George C. Greene and Robert M.

Henry, Langley Research Center, NASA;

Conway B. Leovy and James E. Tillman,

University of Washington; and J. L. Mitchell,

Florida State University

John F. Henz and Vincent R. Scheetz

Radio Station KCOL, Ft. Collins, Colorado

Alan K. Betts, Stephen K. Cox, and Edward J.

Zipser

1978 Morris Tepper

Detachment 4, 7th Weather Wing, Air Weather

Service, Altus Air Force Base, Oklahoma

1979 Robert B. Rice

Guy H. Gray, Jr., Benjamin Brown, William C.

Henry, John E. Michener, and Leon Schirn

1981 National Weather Service Office, Wichita Falls, Texas

1983 Mauna Loa Observatory, NOAA, Mauna Loa, Hilo, Hawaii

1984 Henry T. Harrison

1985 Eric Sloane

George P. Wadsworth and Joseph G. Bryan

1986 J. Murray Mitchell, Jr.

1987 Leonard W. Snellman

1988 Jack C. Thompson

1989 Robert W. Burpee and Leonard W. Snellman

Joseph O. Fletcher 
Henry Lansford

1991

1992

Frederick Sanders

Ira W. Geer

Harry Van Loon

1993 Daniel L. Albritton and Robert T. Watson

Robert W. Corell, J. Michael Hall, and

Shelby G. Tilford

National Hurricane Center, Miami, Florida

Joint Typhoon Warning Center, Guam

Central Pacific Hurricane Center, Honolulu,

Hawaii

National Weather Service Forecast Office,

Miami, Florida

National Weather Service Forecast Office,

New Orleans, Louisiana

National Weather Service Office, Lihue

Bryan Norcross

United States Air Force 1690th Weather Group

(Provisional), Saudi Arabia

Kathleen C. Hale
William H. Hooke

Edwin F. Danielsen

Alfred H. Woodcock

Roger B. Lukas and Peter J. Webster

John R. Christy and Roy W. Spencer

The National Weather Service Spaceflight

Meteorology Group

James F. W. Purdom, Dennis Chesters, and

W. Paul Menzel

Christopher S. Velden

Brian E. Doty

Edward J. Hopkins

NCEP-NCAR Reanalysis Project

2001 Mary Ann Cooper, "for outstanding work on the medical effects of lightning, which has enhanced the treatment of lightning strike victims and revolutionized lightning safety worldwide."

Great Lakes Forecasting System: Keith W. Bedford, director; David J. Schwab, director; Yifei Philip Chu; Brendon Hoch; John G. W. Kelley; Chih Feng Kuan; Sean O’Neil; David P. Podber; Panagiotis Velissariou; David J. S. Welsh; Chieh-Cheng James Yen; Jay S. Hobgood; and Carolyn J. Merry, "for developing the first U.S. coastal forecasting system to make routine operational predictions of currents, temperatures, and key trace constituents."

NWS Forecast Office, Norman, Oklahoma; Oklahoma Department of Public Safety; KFOR, Channel 4; KOCO, Channel 5; KWTV, Channel 9; Southwest Independent Repeater Association; and Oklahoma Climatological Survey, "for outstanding and well-coordinated actions, before, during and after the historic 3 May 1999 tornado outbreak in central Oklahoma, which prevented untold deaths and minimized the impact of the devastating storms."

\section{Louis J. Battan Author's Award}

\begin{tabular}{|c|c|c|c|c|c|}
\hline 1989 & Craig F. Bohren & 1995 & Edward N. Lorenz & 1998 & Zbigniew Sorbjan \\
\hline 1990 & Stephen H. Schneider & 1996 & Thomas E. Graedel and & 1999 & Thomas J. Kierein and \\
\hline 1992 & John W. Firor & & Paul J. Crutzen & & John Buxton \\
\hline 1993 & Robert Marc Friedman & 1997 & Jack Fishman and & 2000 & Richard C. J. Somerville \\
\hline 1994 & Jack Williams & & Robert Kalish & & \\
\hline
\end{tabular}

2001 Howard B. Bluestein, "for Tornado Alley: Monster Storms of the Great Plains, a fascinating story about thunderstorms on the Great Plains, their formation and evolution, and the people who study them." 
The Banner I. Miller Award

$\begin{array}{ll}1977 & \text { Charles J. Neumann and } \\ & \text { Miles B. Lawrence } \\ 1979 & \text { Lloyd Shapiro } \\ 1981 & \text { William M. Frank } \\ 1982 & \text { James C. Sadler } \\ 1984 & \text { Yoshio Kurihara and } \\ & \text { Robert E. Tuleya } \\ 1985 & \text { Greg J. Holland }\end{array}$

1986
1989
1992
1994

Mark DeMaria

Mark DeMaria

Kerry A. Emanuel and

Richard Rotunno

William M. Gray,

Christopher W. Landsea,

Paul W. Mielke, Jr., and

Kenneth J. Berry
1995 Thomas A. Guinn and
Wayne H. Schubert
1998 Morris A. Bender,
Rebecca J. Ross,
Robert E. Tuleya, and
Yoshio Kurihara
1999 John E. Molinari

2001 Christopher S. Velden, Timothy Olander, Steve Wanzong, and Raymond M. Zehr, "for two outstanding contributions on satellite techniques that address tropical cyclone intensity and track prediction problems published during the years 1996-99: 'The impact of multispectral GOES-8 wind information on Atlantic tropical cyclone track forecasts in 1995. Part I: Dataset methodology, description, and case analysis' (MWR, Vol. 126) and 'Development of an objective scheme to estimate tropical cyclone intensity from digital geostationary infrared imagery' (WAF, Vol. 13).”

\section{The Robert Leviton Award}
1987
Gregory Byrne
1994
Igor Gonta
1998 Seiji Kato
1990 Haflidi H. Jonsson
1996 Scott J. Richardson
2000 Alison W. Grimsdell

2001 Raymond A. Shaw, "for his paper on the development of an electrodynamic levitation system to study individual cloud particles typically found in the upper troposphere."

\section{The Max A. Eaton Prize}

\begin{tabular}{|c|c|c|c|c|c|}
\hline \multirow[t]{2}{*}{1976} & Steven Payne and & 1982 & Mark DeMaria & 1992 & Christopher W. Landsea \\
\hline & Mary M. McGarry & & Greg J. Holland & 1994 & Elizabeth A. Ritchie \\
\hline \multirow[t]{3}{*}{1977} & Albert G. Boulanger and & 1984 & John R. Anderson & 1995 & J. Dominique Möller \\
\hline & Michael W. Maier & & Robert T. Merrill & & Randall J. Kallenbach \\
\hline & Clifford A. Mass & 1985 & V. Mohan Karyampudi & 1998 & Anantha Aiyyer \\
\hline 1979 & Peter R. Bannon & 1987 & Candice Weatherford & & Arlene G. Laing \\
\hline 1981 & Edwin Nuñez & 1989 & Jong-Jin Baik & 2000 & Katherine A. Harris \\
\hline
\end{tabular}

2001 James P. Kossin, "for his paper, 'Observational Evidence for Horizontal Mixing in the Hurricane NearCore'."

\section{The Spiros G. Geotis Prize}

1996 Lawrence D. Carey

1998

Sabine Göke

2000

Eszter J. Barthazy 
The Father James B. Macelwane Annual Awards in Meteorology

1960 William E. Shenk (First

Prize)

Joel Myers (Second

Prize)

Francis P. W. Ho (Third

Prize)

1963 John B. Armstrong (First

Prize)

Francis W. Lemire

(Second Prize)

1964 Charles B. Pyke (First

Prize)

Marlies Oberlander

(Second Prize)

Walter A. Lyons (Third

Prize)

Edward E. Hindman II

(Honorable Mention)

1965 William L. Woodley

(First Prize)

John R. Dickson (Second

Prize)

Edward E. Hindman II

(Third Prize)

Joseph Hilary Golden

(Honorable Mention)

1966 Edward E. Hindman II

(First Prize)

Ann E. Gargett (Second

Prize)

D. W. Hartlieb (Third

Prize)

1968 Peter H. Hildebrand

(First Prize)

Andrew J. Heymsfield

(Second Prize)

1969 I. R. Graham (First Prize)

Victor F. Morris (Second

Prize)

Andrew J. Heymsfield

(Third Prize)

1970 Andrew J. Heymsfield

(First Prize)

Carlyle H. Wash (Second

Prize)

Boris Weisman (Third

Prize)

1971 Dean G. Duffy (First

Prize)

Sharon E. Nicholson

(Second Prize)
1972 Robert M. Friedman

(First Prize)

1973 Louis W. Uccellini (First

Prize)

Mary Ann Hrivnak

(Second Prize)

Douglas Beadle (Third

Prize)

1974 Robert M. Thompson, Jr. (First Prize)

David B. Gilhousen

(Second Prize)

1975 Paul W. Greiman (First

Prize)

John S. Jensenius, Jr.

(Second Prize)

1976 Stephen J. Colucci (First

Prize)

Susan A. Ducey (Second

Prize)

Nancy E. Westcott

(Third Prize)

1977 Jeffrey D. Hawkins (First

Prize)

David Schachterle

(Second Prize)

1978 Paul E. Ciesielski (First

Prize)

Frederick E. Brennan

(Second Prize)

Kevin R. Knupp (Second

Prize)

1979 John R. Lanzante (First

Prize)

Daniel C. David (Second

Prize)

1980 Jay W. Schlegel and

Gerard K. Butch

(First Prize)

Kevin A. Mundell

(Second Prize)

1981 Patricia M. Dare (First

Prize)

Christian J. Grund

(Second Prize)

1982 Keith W. Dixon (First

Prize)

Alan S. Tetenbaum

(Second Prize)

1983 David M. Legler (First

Prize)

Paul J. Meyer (Second

Prize)
1984 Paul G. Wolyn (First

Prize)

Barbara A. Chance

(Second Prize)

Richard A. Fulton

(Second Prize)

1985 Craig R. Burfeind (First

Prize)

Jeffrey S. Tilley (Second

Prize)

1986 Wendy E. Schreiber

(First Prize)

Jose D. Fuentes (Second

Prize)

Luther A. Carroll III

(Third Prize)

1987 Haig Iskenderian (First

Place)

Christopher W. Landsea

(Second Place)

J. Todd Hawes (Third

Place)

Brad W. Orr (Third

Place)

John W. Conway

(Honorable Mention)

1988

Anton Seimon (First

Place)

Douglas K. Miller

(Second Place)

1989

Renee A. Pruett (First

Place)

Jay P. Breidenbach

(Second Place)

1990

Maribeth Stolzenburg

(First Place)

Gregory J. Hakim

(Second Place)

Bruce B. Smith (Third

Place)

1991 Richard D. Knabb (First

Place)

Gregory J. Hakim

(Second Place)

Russell S. McCormack

(Third Place)

Douglas A. Speheger

(Third Place)

Shane D. Mayor (Honor-

able Mention) 
1992 David M. Babb (First

Place)

Robert A. Velasco

(Second Place)

Joseph Paluszek (Third

Place)

Robert F. Rogers (Third

Place)

1993 Susan S. Minton (First

Place)

Thomas M. Renkevens

(Second Place)

David R. Stettner (Third

Place)
1994 David L. Montroy (First

Place)

Todd A. Hutchinson

(Second Place)

Susan L. Howard (Third

Place)

1995 Greg E. Mann (First

Place)

Michael W. Gleeson

(Second Place)

Michael A. Bell (Third

Place)

Todd S. Richards

(Honorable Mention)
1996 Daniel P. Guertin (First

Place)

Timothy M. Winter

(Second Place)

1997 Matthew D. Eastin (First

Place)

Paul M. Markowski

(Second Place)

Brian A. Cosgrove

(Third Place)

1998 Mark C. Bove

1999 Jeremy Moore

2000 James E. Coleman

2001 Todd Lericos, The Florida State University, "for his paper, 'Warm Season Lightning Distributions over the Florida Peninsula as Related to Synoptic Patterns."”

\section{The Howard T. Orville Scholarship in Meteorology}

$\begin{array}{lllll}1965 & \text { Daniel L. Smith } & 1979 & \text { Ellen M. Salmon } & 1990 \\ 1966 & \text { James P. Charba } & 1980 & \text { Patricia M. Dare } & 1991 \\ 1967 & \text { Robert L. Gall } & 1981 & \text { Andrew J. Spry } & 1992 \\ 1970 & \text { Allen C. Dittenhoefer } & 1982 & \text { Linda M. Berens } & 1993 \\ 1971 & \text { Cary B. Schudy } & 1983 & \text { Barbara A. Chance } & 1994 \\ 1972 & \text { Lawrence J. Lewis } & 1984 & \text { Michael I. Biggerstaff } & 1995 \\ 1973 & \text { John A. Pollack } & 1985 & \text { Stephen Jascourt } & 1996 \\ 1974 & \text { John A. Toth } & 1986 & \text { Claire A. Woolley } & 1997 \\ 1975 & \text { Daniel Keyser } & 1987 & \text { Douglas K. Miller } & 1998 \\ 1976 & \text { Judith A. Todd } & 1988 & \text { Juliana G. Minkowitz } & 1999 \\ 1977 & \text { Bradley R. Colman } & 1989 & \text { Stephen J. Vavrus } & 2000 \\ 1978 & \text { Leo J. Donner } & & & \end{array}$

2001 Christopher P. Woods, Cornell University

\section{The Howard H. Hanks, Jr., Scholarship in Meteorology}

\begin{tabular}{|c|c|c|c|c|c|}
\hline 1973 & Robert M. Thompson, Jr. & 1984 & Robert X. Black III and & 1992 & Donald M. Rolfson \\
\hline 1974 & Daniel Keyser & & Miriam Blaskovich & 1993 & Jeffrey A. DeToro \\
\hline 1975 & David L. Harper & 1985 & Pamela Camara & 1994 & Matthew E. Pyle \\
\hline 1976 & David D. Adamec & & Timothy J. Schott & 1995 & Gregory E. Gahrs \\
\hline 1977 & Rae Ann Eversole & 1986 & Jonathan E. Martin & & Sara A. Michelson \\
\hline 1978 & Richard C. Deininger & 1987 & William A. Gallus, Jr. & 1996 & Michael M. Whitney \\
\hline 1979 & Dorothea G. Nase & 1988 & Russell E. Treadon, Jr. & 1997 & Kevin D. Dalton \\
\hline 1980 & Robert T. Merrill & 1989 & Scott A. Braun & 1998 & Alana M. Althaus \\
\hline 1981 & Kenneth D. Walker & 1990 & Megan McKay & 1999 & Michele D. Balcom \\
\hline 1982 & Kathy A. Perbeck & 1991 & David A. Waite & 2000 & Grady Dixon \\
\hline 1983 & Richard A. Fulton & & & & \\
\hline
\end{tabular}

2001 Martha R. Stevens, University of Wisconsin-Madison 
The Paul H. Kutschenreuter Scholarship

$\begin{array}{llllll}1992 & \text { Geoffrey D. Considine } & 1995 & \text { James R. Winterle } & 1998 & \text { Daniel S. Cohan } \\ 1993 & \text { Ernest R. Toracinta } & 1996 & \text { Cristina A. Kaufman } & 1999 & \text { Jessica D. Lundquist } \\ 1994 & \text { Shannon E. White } & 1997 & \text { Keith K. Contre } & 2000 & \text { Jessica M. Kleiss }\end{array}$

2001 Benjamin J. Miriovsky, University of Nebraska—Lincoln

\section{The Dr. Pedro Grau Undergraduate Scholarship}

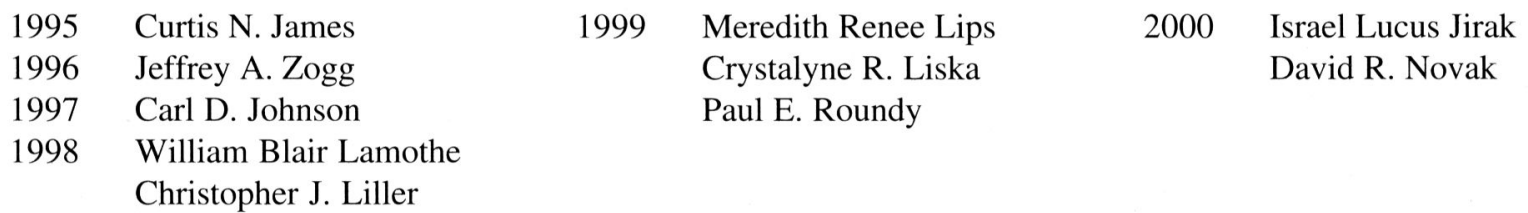

2001 Samuel B. Ajayi, University of Texas-Austin

Emily C. Kowalski, Valparaiso University

\section{The Guillermo Salazar Rodriguez Undergraduate Scholarship}

$\begin{array}{ll}2000 & \text { Robyn Lynn Shirk } \\ \text { Jonathan L. Vigh }\end{array}$

2001 Keith M. Weber, University of Kansas

Mark C. Willis, The Florida State University

\section{The AMS 75th Anniversary Scholarship}

\begin{tabular}{|c|c|c|c|}
\hline Julie M. Caron & 1997 & Alison Gillis & \\
\hline Joseph A. MacDonald & 1998 & Michael J. Wilson & \\
\hline
\end{tabular}

2001 Carl H. Tape, Carlton College

\section{The Mark J. Schroeder Scholarship in Meteorology}
1997 Diana Lynn Sorensen
1999 Scott M. Steiger
2000
Stephen R. Hudson
1998 Marc Jason Weinberg
2001 Robert J. Berg, North Carolina State University

\section{The Richard and Helen Hagemeyer Scholarship}
1997 Brett Weston Newkirk
1999 Kyle A. Beatty
2000
Steven C. Decker
1998 Callie Lynn Buchanan

2001 Anneliese C. Sherer, The Pennsylvania State University 
2000 Jason Arvid Otkin

2001 Tracy L. McCormick, Lyndon State College

\section{AMS/Industry Minority Scholarships}

$\begin{array}{ll}1993 & \text { Martin J. Cole } \\ & \text { Shani M. Phillips } \\ & \text { Hubert C. Ho } \\ & \text { Violeta Moldonado } \\ & \text { Amy M. Tolliver } \\ & \text { Jeremy B. Alexander } \\ & \text { Errol L. Brown } \\ & \text { Janessa C. Cobb } \\ & \text { Heather A. Elenbaas } \\ & \text { Enrique E. Urueta II (AMS/Weather Channel) } \\ & \text { David J. Williams } \\ & \text { Angela J. Yu } \\ & \text { Mindy A. Chavez } \\ & \text { Thomas Ferede } \\ & \text { Bradley C. Navarro } \\ & \text { Aaron S. Pratt (AMS/Weather Channel) } \\ \text { Anthony J. Torres } \\ \text { Adam R. Abate } \\ \text { Wendy L. Bleyl } \\ \text { Elita Y. Cheung (AMS/Weather Channel) } \\ \text { Angela C. Hughes } \\ \text { Jeffrey P. Lowther (AMS/Hughes Santa } \\ \text { Barbara Remote Sensing) } \\ \text { Stacy A. McCallan (AMS/Computer Sciences } \\ \text { Corporation) } \\ \text { Anthony Nguyen (AMS/PRC Inc.) }\end{array}$

1999 Wesley C. Acosta (AMS/National Environmental Satellite, Data, Information Service)

Tara L. Arness

Lindsay G. Burge

Daniel J. Fuentes (Larry R. Johnson Memorial Minority Scholarship)

Russell L. Hudgins

Paul M. Iniguez (AMS/Computer Sciences Corporation)

Megan L. Rock (AMS/National Environmental Satellite, Data, Information Service)

Remeisha D. Shade (AMS/Weather Channel)

Erika M. Sharp (AMS/Raytheon Santa Barbara Remote Sensing)

2000 Ramya Ananthanarayanan (Computer Science Corp.)

Lanchi D. Huynh (AMS Programs in Support of Science and Education)

James P. Lowther (AMS Programs in Support of Science and Education)

Paulo R. Molina (AMS Programs in Support of Science and Education)

Brandon L. Noriega (The Weather Channel)

Jonathan M. Pacheco (The Larry R. Johnson Memorial Minority Scholarship)

Ashton D. Robinson-Cook (The Robert T. Ryan Scholarship)

Scott T. Williams (AMS Programs in Support of Science and Education) Joyce G. Wang (AMS Programs in Support of Science and Education)

Ariel Rodriguez (Climadata Corp)

2001 Erik J. Adame, University of Oneota (IBM)

Kyle T. Bush, Allegheny College (AMS)

Gabriel S. Garfield, University of Oklahoma (RGII Technologies)

Abigail K. Hicks, St. Mary’s University (The Weather Channel ${ }^{\circledR}$ )

Marcia T. Hsu, University of Georgia (AMS)

Julie L. Johnston, Univ. of Nevada-Las Vegas (AMS) 
Vianney Lopez, Cornell University (ClimaData)

Jordan E. Lucero, The Florida State University (Litton PRC)

Justin J. Mosely, The Florida State University (NBC-TV/Bob Ryan)

Ronald K. Porter, Eckerd College (RS Information Systems)

Orlando Segura Jr., Harvard University (Raytheon Santa Barbara)

Jia Jun (Jason) Wen, Harvard University (Computer Science Corporation)

\section{AMS/Industry/Government Graduate Fellowships}

1991

Brian A. Colle (Computer Sciences Corporation)

Caryn L. Dean (GE Astro Space Division)

Robert Jacob (Cray Research, Inc.)

Stephen E. Marshall (PRC Inc.)

James A. Shepard (TRW Foundation)

1992 David M. Babb (PRC Inc.)

Andrew Humphrey (Cray Research, Inc.)

Kelly McNerney (Computer Sciences Corporation)

Martin Murphy (Space Systems/Loral)

Christopher Plouff (TRW Foundation)

George Pouliot (Hughes Information Technology Company)

Robert L. M. Rausch (Paramax Systems Corporation)

John Schrage (ITT Aerospace Communications Division)

James Turner (NOAA's Office of Global Programs)

1993 Mark A. Askelson (Cray Research, Inc.)

Kevin C. Baggett (PRC Inc.)

Kyle W. Blasch (TRW Foundation)

Gregory M. Fall (ITT Aerospace Communications Division)

M. Thomas Filiaggi (Computer Sciences Corporation)

H. David Gold (Hughes Information Technology Company)

David L. Montroy (NOAA's Office of Global Programs)

David S. Myers (NOAA's Office of Global Programs)

John J. Persing (Martin Marietta Astro Space)

Raymond A. Shaw (Paramax Systems Corporation)

Scott C. Sheridan (Space Systems/Loral)
1994 Bruce T. Anderson (Space Systems/Loral)

Eyad H. Atallah (GTE's Federal Systems Division)

Jerald A. Brotzge (Unisys Corporation, Government Systems Group)

Kathleen A. Edwards (NOAA's Office of Global Programs)

Eric R. Hilgendorf (ITT Aerospace Communications Division)

Robert H. Kent (NOAA's Office of Global Programs)

Brett E. McDonald (Cray Research, Inc.)

John J. Mewes (Martin Marietta Astro Space)

Brian D. Skinner (Hughes Information Technology Company)

Jason Stiff (PRC Inc.)

Sharon L. Sullivan (American Meteorological Society)

1995 S. Traci Arthur (GTE's Federal Systems Division)

Corinne M. Carter (American Meteorological Society)

Jonathan L. Case (PRC Inc.)

Stephen G. Gaddy (Loral Defense SystemsEast)

Gregory E. Gahrs (Martin Marietta Astro Space)

Gregory J. Gemmen (Cray Research, Inc.)

Christopher T. Hudgin (Hughes Information Technology Corporation)

Dana E. Lane (NOAA's Office of Global Programs)

Timothy J. Lang (Space Systems/Loral)

Edward A. Mlodzik (ITT Aerospace/ Communications Division)

Phillipe Rolland (DOE Atmospheric Radiation Measurement Program)

James R. Winterle (NOAA's Office of Global Programs) 
Brian M. Astifan (Hughes Information Technology Corporation)

George H. Bryan (National Weather Service)

Matthew T. Carr (American Meteorological Society)

David A. Faysash (Space Systems/Loral)

Cristina A. Kaufman (Cray Research, Inc.)

Greg C. Liknes (DOE Atmospheric Radiation Measurement Program)

Joseph A. MacDonald (NOAA's Office of Global Programs)

Paul M. Markowski (GTE Government Systems Corporation, Federal Systems Division)

Tracy A. Monegan (NOAA's Office of Global Programs)

Matthew D. Parker (ITT Aerospace/Communications Division)

Mark J. Potosnak (Lockheed Martin Astro Space)

Jeffrey M. Warner (PRC Inc.)

1997

Michael C. Coniglio (National Weather Service)

Keith K. Contre (NOAA's Office of Global Programs)

Andrew R. Dean (SGI/Cray Research, Inc.)

Michael A. Kistler (Lockheed Martin Astro Space)

Ian P. Kraucunas (GTE Government Systems)

William G. Lawson (Hughes Information Technology Systems)

Richard J. Lynn (NASA Mission to Planet Earth)

Patrice A. Melancon (NASA Mission to Planet Earth)

Michael A. Mischna (Space Systems/Loral)

Brett W. Newkirk (DOE Atmospheric Radiation Measurement Program)

Payal P. Parekh (NOAA's Office of Global Programs)

Stephen G. Strum (ITT Aerospace/Communications Division)

Christopher C. Weiss (NASA Mission to Planet Earth)

1998 Amanda S. Adams (DOE Atmospheric Radiation Measurement Program)

Eric S. Blake (NASA Mission to Planet Earth)

Robert W. Carver (National Weather Service)

Scott J. Eichelberger (NOAA's Office of Global Programs)

Joanne M. Feldman (GTE Government Systems)
Chris A. Fiebrich (National Science Foundation Division of Atmospheric Sciences)

Michael R. Hiscock (NASA Mission to Planet Earth)

Jeanne M. Jagodzinski (NASA Mission to Planet Earth)

Erica M. Loechl (ITT Aerospace/Communications Division)

Nathan D. Marsili (NASA Mission to Planet Earth)

Jeremy L. Moore (American Meteorological Society)

Elizabeth A. Northcott (National Science Foundation Division of Atmospheric Sciences)

Jeffrey S. Olsen (Systems Space/Loral)

David A. Tucker (DOE Atmospheric Radiation Measurement Program)

Shawn M. Weagle (Silicon Graphics/Cray Research)

Marc J. Weinberg (Raytheon Information Systems Company)

Michael M. Whitney (NOAA's Office of Global Programs)

Shane E. Wood (National Science Foundation Division of Atmospheric Sciences)

1999 Curtis R. Alexander (National Weather Service)

Michele D. Balcom (National Science Foundation Division of Atmospheric Sciences)

Kyle A. Beatty (National Science Foundation Division of Atmospheric Sciences)

Howard I. Berger (Lockheed Martin Missiles \& Space)

Gregory J. Braun (DOE Atmospheric Radiation Measurement Program)

Kevin P. Connors (Office of Naval Research)

Justin A. Cox (ITT Industries Aerospace/ Communications Division)

Julie L. Demuth (GTE Government Systems)

Jill C. Derby (NOAA's Office of Global Programs)

Francesco Fiondella (Space Systems/Loral)

Andrea L. Glaza (American Meteorological Society)

John M. Haynes (NOAA's Office of Global Programs)

Matthew J. Hostutler (Raytheon Systems Company)

Daniel R. Jamieson (NASA's Earth Science Enterprise)

Daniel T. Lindsey (National Science Foundation Division of Atmospheric Sciences) 
Crystaline R. Liska (Silicon Graphics, Inc.)

Christopher M. McAloon (NASA's Earth

Science Enterprise)

Brian P. Reen (Office of Naval Research)

Paul E. Roundy (DOE Atmospheric Radiation Measurement Program)

John E. Strack (NASA's Earth Science Enterprise)

Elizabeth C. Wood (NASA's Earth Science Enterprise)

Wynn W. Yin (Hughes Space and Communications Company)

2000 Anthony Arguez (DynCorp)

Christopher D. Cappa (DOE Atmospheric Radiation Measurement Program)

Kimberly A. Elkins, University of California-Berkeley (National Science Foundation Division of Atmospheric Sciences)

Scott E. Giangrande (National Weather Service)

Alicia Jillian Hardy (Office of Naval Research)

Stephen R. Hudson (DOE Atmospheric Radiation Measurement Program)

Daniel M. Matross (National Science Foundation Division of Atmospheric Sciences)
Sergey L. Napelenok (NOAA'S Office of Global Programs)

Wesley D. Terwey (National Science Foundation Division of Atmospheric Science)

Sarah A. Tessendorf (ITT Industries Aerospace/Communications Division)

Blaine C. Thomas (Lockheed Martin Missiles \& Space)

Barbara E. Tobey (NASA'S Earth Science Enterprise)

Jonathan L. Vigh (IBM)

Michael R. Wagg (American Meteorological Society)

Cory A. Wolff (NASA'S Earth Science Foundation)

Kyle A. Hilburn (NASA'S Earth Science Enterprise)

Virginia A. Chadwick (NASA'S Earth Science Enterprise)

Cassie M. Archuleta (NOAA'S Office of Global Programs)

Michael D. Hurwitz (Office of Naval Research)

2001 Robert J. Berg, University of Miami (Office of Naval Research)

Russell J. Chibe, Colorado State University (ITT Industries Aerospace/Communications Division)

Tracy K. Depue, Colorado State University (NASA's Earth Science Enterprise)

Meagan J. Eagle, Stanford University (AMS)

Jeffrey W. Frame, The Pennsylvania State University (National Weather Service)

Matthew J. Haugland, University of Oklahoma (NASA's Earth Science Enterprise)

Rynda C. Hudman, Harvard University (NOAA’s Office of Global Programs)

Daryl T. Kleist, University of Wisconsin-Madison (NASA's Earth Science Enterprise)

Michael D. Knepp, North Carolina State University (National Science Foundation Division of Atmospheric Sciences)

Tracy L. McCormick, North Carolina State University (NOAA’s Office of Global Programs)

Benjamin J. Miriovsky, University of Iowa (National Science Foundation Division of Atmospheric Sciences) 
Hanne V. Murphey, University of California-Los Angeles (National Science Foundation Division of Atmospheric Programs)

Andrea B. Saunders, Colorado State University (Office of Naval Research)

Russ S. Schumacher, Colorado State University (IBM)

Anneliese C. Sherer, University of Oklahoma (Subaru)

Martha R. Stevens, University of Washington (DOE Atmospheric Radiation Measurement Program)

Stephanie A. Stroman, University of Oklahoma (NASA's Earth Science Enterprise)

Andrew A. Taylor, University of Oklahoma (DOE Atmospheric Radiation Measurement Program)

\section{The AMS Graduate Fellowship in the History of Science}
1999
Gregory T. Cushman
2000
Kristine C. Harper

2001 John A. C. Wedge, University of Illinois

\section{AMS/Industry Undergraduate Scholarships}

1991 Stephanie M. Blozy (Qualimetrics, Inc.)

1992 Kathleen Eyerman (Qualimetrics, Inc.)

Sharon Hauer (Hughes STX Corporation)

Joseph Rohrbach (AER Inc.)

Brian Skinner (Aeromet Inc.)

Tina Johnson (Science and Technology

Corporation)

John Mewes (Campbell Scientific, Inc.)

Jerald Brotzge (Atmospherics, Inc.)

1993 Jason J. Levit (Aeromet Inc.)

Stephen G. Gaddy (Hughes STX Corporation)

Christopher T. Hudgin (Science and Technology Corporation)

Jonathan L. Case (Alden Electronics, Inc.)

Kathryn Klawiter (Radian Corporation)

Kevin A. Cooper (Handar Inc.)

Jessica L. Neu (The MITRE Corporation)

Cristina A. Kaufman (National Council of Meteorologists)

John J. Rozbicki (Vaisala, Inc.)

Teresa A. Grabler (Concurrent Computer Corporation)

Jeremy A. Marzani (AAI Systems Management Incorporated)

1994 David A. Ahijevych (Atmospheric and Environmental Research, Inc.)

Mark J. Gibbas (Concurrent Computer Corporation)
Julie R. Givan (Enterprise Electronics Corporation)

Sonya C. Kulkarni (Science and Technology Corporation)

Daniel F. Lara (Campbell Scientific, Inc.)

Greg C. Liknes (The MITRE Corporation)

Brian P. Mackey (WSI Corporation)

Douglas E. Murphy (Surface Systems, Inc.)

Noah M. Nigg (R. M. Young Company)

Ryan J. Sharp (AAI Systems

Management Inc.)

Ryan J. Zerr (Hughes STX Corporation)

1995 Michael T. Batdorf (Handar, Inc.)

Joseph Parks Camp (Science and Technology Corporation)

Adam S. Cohen (Vaisala, Inc.)

Michael A. Kistler (AAI Systems

Management Inc.)

W. Gregory Lawson (Belfort Instrument Co.)

Timothy S. Martin (Particle Measuring

Systems, Inc.)

John V. Matthews (EARTH TECH)

Michael A. Mischna (Alden Electronics, Inc.)

CarrieAnn Ortolani (The MITRE

Corporation)

Jon D. Radakovich (Hughes STX

Corporation)

Stephen G. Strum (Radian Corporation) 
Jay-Calvin Uyemura-Reyes (Nat'1 Council of Industrial Meteorologists)

Joel C. Widenor (Concurrent Computer Corporation)

Shane E. Wood (Office of the Federal Coordinator for Meteorology)

1996 Amy E. Blackmore (Surface Systems, Inc.)

Robert W. Carver (Particle Measuring Systems, Inc.)

Donna M. Couture (Office of the Federal Coordinator for Meteorology)

Michael A. Dente (Hughes STX Corporation)

Dora Farkas (Atmospheric and

Environmental Research, Inc.)

Shawn R. Ferreria (Campbell Scientific, Inc.)

Cynthia R. Knapp (Kavouras Inc.)

Ian P. McAbeer (R. M. Young Company)

Jeremy L. Moore (Science and Technology Corporation)

Robert E. Moyer IV (AAI Systems Management Incorporated)

Avanti J. Rao (Concurrent Computer Corporation)

Ryan L. Solomon (Enterprise Electronics Corporation)

1997 Mohan B. Dangi (Particle Measuring Systems, Inc.)

Jill C. Derby (AAI Systems Management Incorporated)

Brian C. Dicola (Concurrent Computer Corporation)

Cory A. Fulton (Handar, Inc.)

Ryan P. Girard (Vaisala, Inc.)

John M. Haynes (Accuweather, Inc.)

Matthew J. Hostutler (SAIC, Center for Atmospheric Physics)

Tim L. Kramer (Science and Technology Corporation)

Daniel T. Lindsey (Enterprise Electronics Corporation)

Janet E. Martinez (Radian Electronics Division)

Matthew E. Newman (Nat'l Council of Industrial Meteorologists)

Nicondra T. Norwood (Office of the Federal Coordinator for Meteorology)

Brian P. Reen (EARTH TECH)

Scott M. Steiger (Weather Services International)

Blaine C. Thomas (Belfort Instrument Co.)

Mandy K. Upton (Weathernews Inc.)

Carrie M. Wood (The MITRE Corporation)

David P. Yorty (Hughes STX Corporation)
1998

Jennifer B. Allan (Weather Services Corporation)

Anthony Arguez (Enterprise Electronics Corporation)

Minesh K. Bacarania (SAIC, Center for Atmospheric Physics)

Peggy R. Concannon (R. M. Young Company)

Brett L. Cummins (Baron Services)

Kyle A. Hilburn (Kavouras Inc.)

Connie J. Hissung (Office of the Federal Coordinator for Meteorology)

Benjamen B. Korzeniewski (Science and Technology Corporation)

Rebecca E. Kyle (Radian Electronic Systems)

Hanne V. Murphey (Raytheon STX Corporation)

Pamela L. Pedersen (Atmospheric and Environmental Research, Inc.)

Shelley L. Potts (Solar Light Company)

Scott D. Preston (Systems Management Incorporated)

Tiffany R. Rippeon (Alden Electronics Inc.)

Cory A. Wolff (Campbell Scientific, Inc.)

1999 Lee T. Ringer (Information and Technology Scientific Services)

Mindy L. Bumgarner (Science and Technology Corporation)

Garett J. Argianas (Vaisala Inc.)

Elizabeth A. Robbins (Handar, Inc.)

Joseph E. Lapka (All Weather Incorporated)

Benjamin D. Teolis (Enterprise Electronics Corporation)

Tracy L. McCormick (Weather Services International)

Scott W. Parker (Office of the Federal Coordinator for Meteorology)

Timothy O. Markle (AccuWeather, Inc.)

Bradley C. Navarro (Weathernews Inc.)

Steven L. Aves (SAIC, Center for Atmospheric Physics)

Stephanie A. Stroman (Mahoney Consultants)

2000 Kurt M. Buffalo (Raytheon Information Technology and Scientific Services Division)

Melissa S. Bukovsky (Baron Services)

Daniel T. Dawson (SAIC, Center for Atmospheric Physics)

Jared Hoffman (Atmospheric and Environmental Research, Inc.)

Brandon W. Kerns (R.M. Young Company)

Nettie R. Lake (Solar Light Company)

Matthew P. Makens (General Dynamics) 
Patrick V. Munoz (Enterprise Electronics Corporation)

Joshua M. Palmer (Autometric, Inc.)
Rebecca J. Stanley (Science and Technology Corporation)

Ryan D. Torn (Office of the Federal Coordinator For Meteorology)

Christopher W. Blackey, Plymouth State College (Vaisala)

Shane H. Brodie, Cornell University (Subaru)

Steven M. Cavallo, The Florida State University (Enterprise Electronic Corp.)

Nicholas J. Eckstein, Bowling Green State University (SAIC, Center for Atmospheric Physics)

Lucas M. Harris, Northern Illinois University (Science and Technology Corporation)

Margaret A. Knuth, University of Miami (Raytheon Information Technology \& Scientific Services Division)

Samuel J. O'Connor, Purdue University (AccuWeather)

Michael T. Schwab, University of Northern Colorado (Office of the Federal Coordinator for Meteorology)

Daniel P. Solari, Plymouth State College (Weather Services International) 